\title{
Caderneta de Saúde da Criança: avaliação do preenchimento dos dados sobre gravidez, parto e recém-nascido
}

Child's Health Record: evaluation of pregnancy, birth and neonatal data filling

Lúcia Maria H. F. Goulart', Claudia Regina L. Alves ${ }^{1}$, Maria Regina de A. Viana², Zeína Soares Moulin ${ }^{3}$, Guilherme Augusto A. do Carmo ${ }^{4}$, Juliana Goulart D. da Costa ${ }^{4}$, Juliana Sartorelo C. B. Almeida ${ }^{5}$

\section{Resumo}

Objetivo: Avaliar o preenchimento dos dados sobre gravidez, parto e recém-nascido na Caderneta de Saúde da Criança (CSC) e conhecer a percepção das mães sobre a função deste instrumento em Belo Horizonte, Minas Gerais.

Métodos: Estudo transversal descritivo de 797 CSC de crianças nascidas entre abril e dezembro de 2005 e entregues às mães na maternidade. Foi utilizado protocolo para avaliar as primeiras páginas da CSC por observação direta e feitas duas perguntas às mães. $\mathrm{O}$ preenchimento de cada item foi categorizado como presente, ausente ou incorreto.

Resultados: O nome da criança não estava registrado em $44 \%$ das CSC. O início do pré-natal estava sem preenchimento em $40 \%$ e o número de consultas, em $31 \%$. O tipo de parto estava em branco em $15 \%$ das CSC; a idade gestacional, em 24\%; e o Apgar de cinco minutos, em 23\% dos casos. Peso ao nascer, comprimento e perímetro cefálico não foram informados em 9, 10 e $15 \%$, respectivamente. O campo destinado à orientação para levar o neonato à Unidade Básica de Saúde (UBS) no quinto dia de vida não foi preenchido em $75 \%$. Apenas $33 \%$ das mães receberam explicações sobre a CSC na maternidade. Para 313 mães, a CSC estava relacionada ao acompanhamento do crescimento e/ou desenvolvimento do seu filho.

Conclusões: $\mathrm{O}$ estudo mostrou falhas no preenchimento da CSC, sugerindo necessidade de esforços para que ela cumpra seu papel na promoção da saúde infantil.

1Doutora em Pediatria, professora adjunta do Grupo de Estudos em Atenção Primária do Departamento de Pediatria da Faculdade de Medicina da Universidade Federal de Minas Gerais (UFMG), Belo Horizonte, MG, Brasil

${ }^{2}$ Professora adjunta do Grupo de Estudos em Atenção Primária do Departamento de Pediatria da Faculdade de Medicina da UFMG, Belo Horizonte, MG, Brasil

${ }^{3}$ Professora-assistente do Grupo de Estudos em Atenção Primária do Departamento de Pediatria da Faculdade de Medicina da UFMG, Belo Horizonte, MG, Brasil

${ }^{4}$ Acadêmico, estagiário do Grupo de Estudos em Atenção Primária do Departamento de Pediatria da Faculdade de Medicina da UFMG, Belo Horizonte, MG, Brasil
Palavras-chave: saúde materno-infantil; registros médicos; atenção primária à saúde; promoção da saúde; recémnascido.

\section{Abstract}

Objective: Evaluate pregnancy, birth and neonatal data filling of the Child's Health Record (CHR) and study mother's perception about the function of this tool in Belo Horizonte, Minas Gerais, Brazil.

Methods: This was a cross-sectional descriptive study of 797 CHR of infants born between April and December 2005 and delivered to their mothers soon after birth. A written protocol was used to assess the first pages of the CHR and mothers were asked two questions. Each $\mathrm{CHR}$ item was classified as correctly, incorrectly or not filled in.

Results: The name of the child was not in the CHR in $44 \%$. The first prenatal visit and the number of prenatal visits were absent in respectively 40 and $31 \%$ of the records. The type of birth was not recorded in $15 \%$ of the CHR, as well as the gestational age in $24 \%$ and the Apgar score at five minutes in $23 \%$. The newborns weight, length and head circumference were not informed in 9, 10 and $15 \%$, respectively. The slot with the orientation to take the newborn to the first pediatric visit on the 5 th day of life was blank in $75 \%$. Only $32 \%$ of the mothers received information about the $\mathrm{CHR}$ at the maternity ward. To 313 mothers, the CHR was important to record follow-up of growth and/or development of their child.

${ }^{5}$ Médica do Grupo de Estudos em Atenção Primária do Departamento de Pediatria da Faculdade de Medicina da UFMG, Belo Horizonte, MG, Brasil

Endereço para correspondência:

Lúcia Maria H. F. Goulart

Avenida Alfredo Balena, 190

CEP 30130-100 - Belo Horizonte/MG

E-mail: luciag@medicina.ufmg.br

Recebido em: 22/10/2007

Aprovado em: 21/01/2008 
Conclusions: An important lack of information was noted in the CHR. Efforts should be directed to improve the quality of information in order to enhance children's health promotion.

Key-words: maternal and child health; medical records; primary health care; health promotion; infant, newborn.

\section{Introdução}

A Caderneta de Saúde da Criança (CSC), implantada pelo Ministério da Saúde a partir de 2005 em substituição ao Cartão da Criança, reúne o registro dos mais significativos eventos para a saúde infantil. Além do cartão de vacina, comporta o registro da história obstétrica e neonatal; indicadores de crescimento e desenvolvimento; aspectos importantes da alimentação, como aleitamento materno, uso de sulfato ferroso e vitamina A; dados sobre a saúde bucal, auditiva e visual - além de intercorrências clínicas e orientações para a promoção da saúde e para a prevenção de agravos, como acidentes e violência doméstica.

A CSC é destinada a todos os nascidos em território brasileiro e, por estar ancorada em ações de acompanhamento e promoção da saúde, inclui-se como estratégia privilegiada nas políticas de redução da morbimortalidade infantil. Os primeiros registros sobre o parto, puerpério, informações sobre o recém-nascido e dados de identificação da criança e da sua família devem ser feitos nas maternidades. Após a alta, os registros subseqüentes são efetuados pelos profissionais responsáveis pelo acompanhamento da criança, nos serviços de atenção primária ou em outros serviços eventualmente procurados.

Para que a CSC cumpra seu papel de instrumento de comunicação, vigilância e promoção da saúde infantil, é essencial sua utilização adequada, o que inclui o registro correto e completo das informações, além do diálogo com a família sobre as anotações ali realizadas. A valorização e a apropriação da CSC pela família refletem a maneira como este instrumento é trabalhado nos serviços de saúde. Neste contexto, o presente estudo teve como objetivo avaliar o preenchimento dos campos sobre gravidez, parto e puerpério e informações sobre o recém-nascido na CSC, bem como conhecer a percepção das mães sobre a função deste instrumento.

\section{Métodos}

Neste estudo transversal e descritivo, estudaram-se as CSC de crianças nascidas em Belo Horizonte, Minas Gerais, entre abril e dezembro de 2005, em maternidades públicas, privadas e conveniadas. Foram incluídas apenas as CSC que haviam sido entregues às mães nas maternidades, excluindo-se aquelas entregues nas Unidades Básicas de Saúde (UBS).

A coleta de dados foi realizada no segundo semestre de 2005, por médicos e enfermeiros alunos do Curso de Especialização em Saúde da Família, em 29 UBS localizadas em cinco dos nove distritos sanitários de Belo Horizonte. A Figura 1 mostra os locais da pesquisa e a distribuição das CSC analisadas.

Para a coleta de dados, foram abordadas as mães das crianças elegíveis, captadas por ocasião do comparecimento, à UBS para atendimento aos seus filhos e/ou por busca ativa, em visitas domiciliares. Foi utilizado um questionário que constava de identificação, 49 questões para verificação direta do preenchimento dos campos das primeiras páginas da CSC (nome e informações da criança); dados sobre gravidez, parto e puerpério; informações sobre o recém-nascido. O preenchimento de cada campo foi avaliado, de acordo com as orientações do Manual para Utilização da Caderneta de Saúde da Criança, como correto, incorreto ou ausente ${ }^{(1)}$.

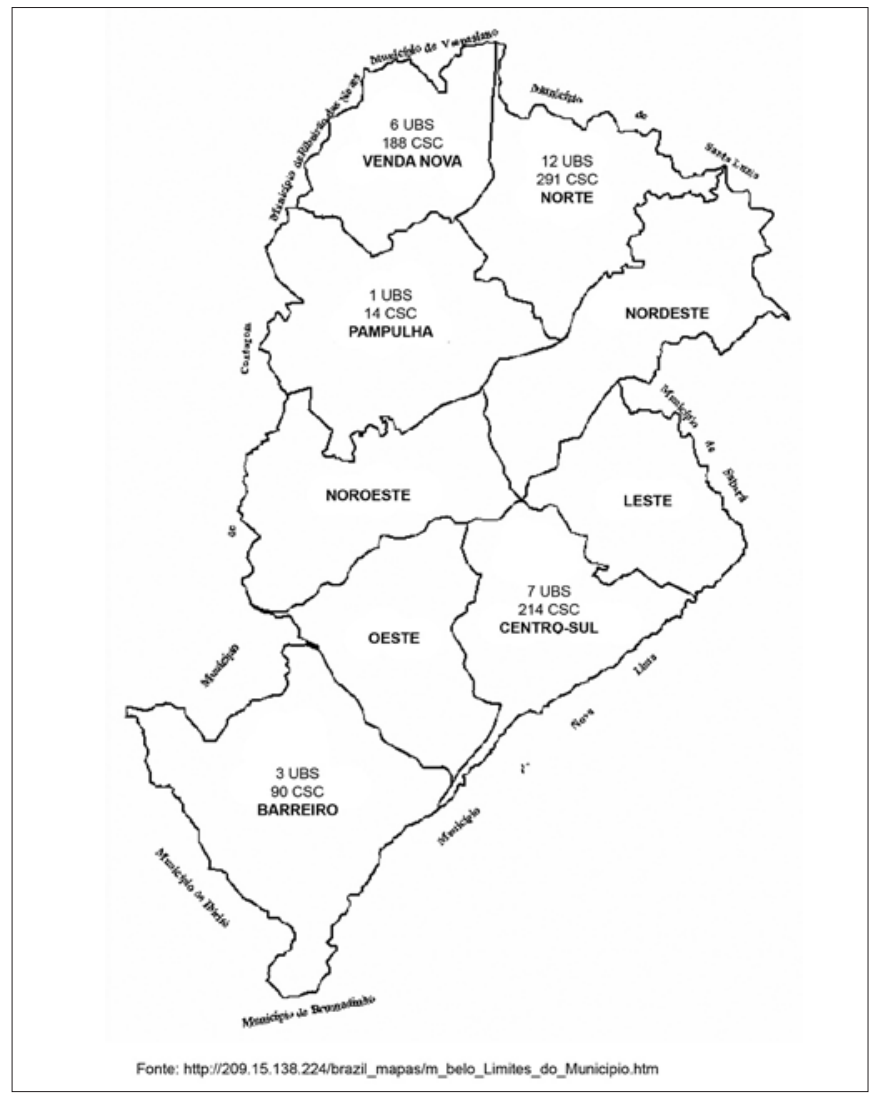

Figura 1 - Distribuição das Carteiras de Saúde da Criança avaliadas por Unidade Básica de Saúde, em cinco das nas nove Regionais de Saúde de Belo Horizonte,Minas Gerais. 
Além da verificação da CSC, foram feitas duas perguntas às mães: "A senhora recebeu explicações sobre esta caderneta na maternidade?" e "Na sua opinião, para que serve a Caderneta de Saúde da Criança?”. As respostas a estas perguntas foram analisadas a partir de classificação por categorias explicativas, identificadas nas falas das próprias entrevistadas.

O software Epi-Info 6.04 foi utilizado para construir e analisar o banco de dados. Os resultados foram expressos em proporção e comparados pelo teste do qui-quadrado, adotando-se o nível de significância de 5\%.

As mães entrevistadas foram esclarecidas sobre os objetivos e métodos da pesquisa e assinaram o termo de consentimento livre e esclarecido. A pesquisa foi aprovada pelo Comitê de Ética em Pesquisa da Secretaria Municipal de Saúde de Belo Horizonte.

\section{Resultados}

Foram estudadas 797 CSC de crianças cuja idade variou de três dias a 8,4 meses, com a mediana de 2,3 meses, nascidas em 17 maternidades de Belo Horizonte (seis públicas), onde receberam a CSC. A maioria dos nascimentos (448, 56\%) havia ocorrido em maternidades privadas/conveniadas com o Sistema Único de Saúde (SUS).

Em todas as CSC foram identificadas falhas no preenchimento - predominantemente campos deixados em branco. Foi encontrada uma pequena proporção de preenchimentos incorretos, sendo o nome da criança, o da mãe e o endereço os itens que apresentaram maior proporção de incorreções no registro (respectivamente em 6,4 e $4 \%$ ). O peso ao nascer foi o campo com melhor preenchimento, sendo registrado corretamente em $91 \%$ das CSC.

A situação do preenchimento dos campos referentes aos dados de identificação da criança na primeira página da CSC é apresentada na Figura 2. O campo referente ao nome da criança estava em branco em $44 \%$ dos casos. A idade média das crianças sem o nome registrado na caderneta foi de 68 dias, sendo a mediana de 59 dias.

A Figura 3 mostra a situação das 797 CSC em relação ao preenchimento dos dados sobre gravidez, parto e puerpério e a Figura 4 ilustra o preenchimento dos campos referentes às condições da criança ao nascer. Não houve diferença significativa entre as maternidades públicas e privadas e/ ou conveniadas em relação ao registro do Apgar de cinco minutos $(p=0,66)$.

Em apenas $25 \%$ das CSC foram anotadas as orientações para a Primeira Semana de Saúde Integral. Em relação a este procedimento, houve diferença entre maternidades públicas e privadas/conveniadas, sendo que as públicas o fizeram com freqüência significantemente maior do que as demais $(p=0,001)$, embora ainda muito baixa (30\%). Verificou-se que 537 (67\%) mães entrevistadas não tinham recebido explicações sobre a CSC na maternidade. Não houve diferença significativa entre as maternidades públicas e privadas e/ou conveniadas em relação ao fornecimento de explicações $(p=0,14)$.

Foram analisadas as respostas à pergunta "para que serve a Caderneta de Saúde da Criança?” de 614 questionários (77\%

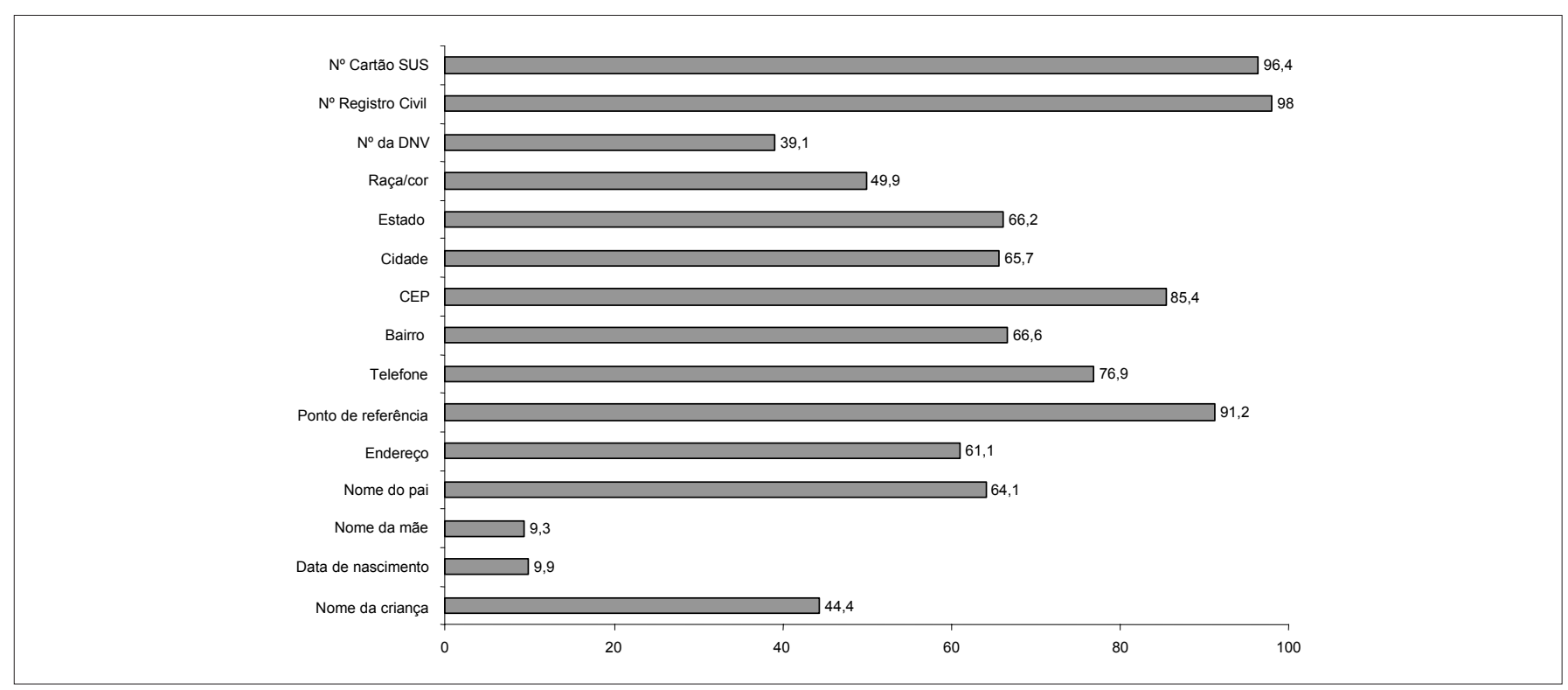

Figura 2 - Porcentagem de não preenchimento dos campos de identificação da criança na CSC, Belo Horizonte, Minas Gerais, $2005(n=797)$. 
do total). Foram excluídos 183 questionários por falta de legibilidade, inconsistência da resposta, ausência da anotação e extravio da folha anexa ao questionário destinada à resposta. Em 313 (51\%), as palavras "acompanhamento" e/ou "crescimento" e/ou "desenvolvimento" foram mencionadas: " $A$ CSC serve para acompanhamento da vida da criança, tanto pela mãe, quanto pelo médico". A CSC serve como cartão de vacina para $45 \%$ das mães, sendo que $2 / 3$ destas relacionaram outras funções, além do registro de vacinas. Para 77 mães (12\%), a
CSC serve como instrumento de informação e ou de comunicação com a família: "Serve para explicar os meses, cada passo da criança". Para 4\%, a CSC serve, além de outras funções, como documento da criança: "É igual à identidade da criança, onde eu for tenho que levar". Para 40 entrevistadas, a CSC "não serve para nada". Entre estas, 37 (92\%) não haviam recebido explicação na maternidade, proporção significativamente maior quando comparada com o grupo de mães que atribuíram pelo menos uma função para a $\operatorname{CSC}(p=0,001)$.

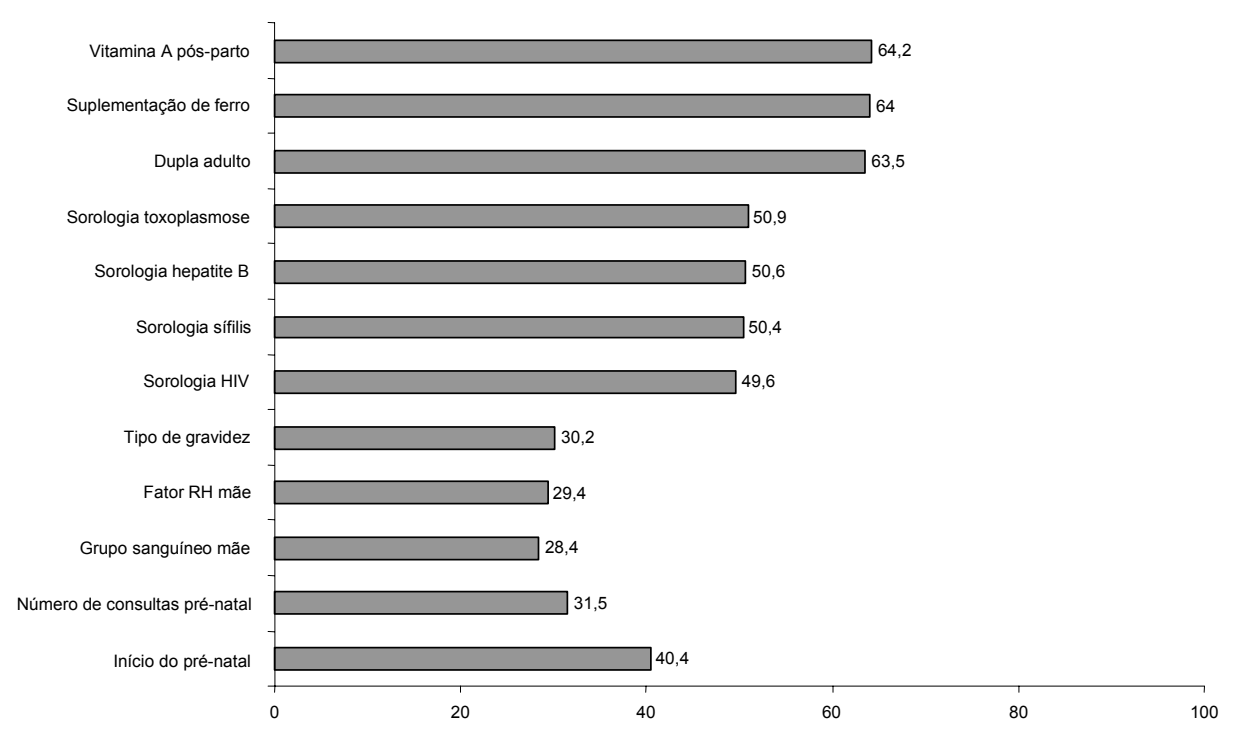

Figura 3 - Porcentagem de não preenchimento dos dados sobre gravidez, parto e puerpério das CSC, Belo Horizonte, Minas Gerais, $2005(n=797)$.

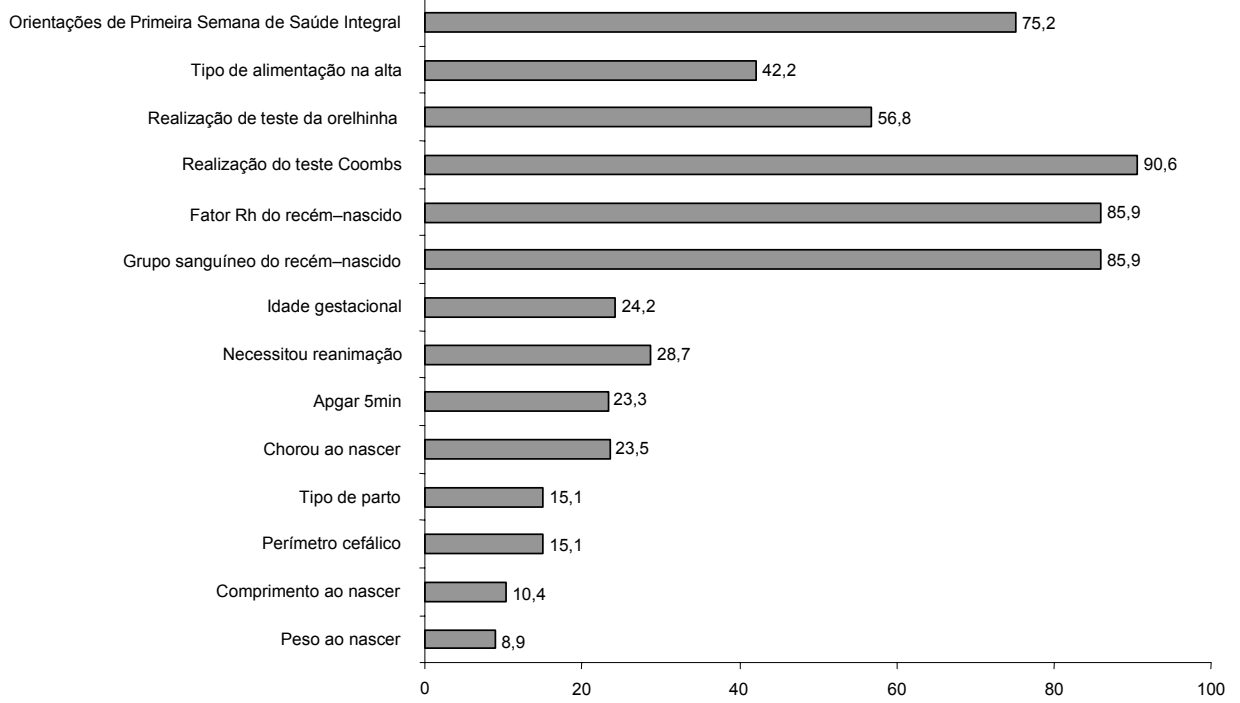

Figura 4 - Porcentagem de não preenchimento das informações sobre as condições do recém-nascido nas CSC, Belo Horizonte, Minas Gerais, 2005 ( $n=797)$. 


\section{Discussão}

Os resultados mostraram a precariedade do preenchimento da CSC. Vários registros importantes foram deixados em branco numa proporção bastante elevada.

Na página destinada à identificação, verificou-se a ausência do nome da criança em $44 \%$, o que poderia ser aceitável em cadernetas de recém-nascidos, pressupondo-se que a família ainda não havia registrado a criança ou o nome ainda não estava definido. Todavia, a média de idade destas "crianças sem nome" foi de 68 dias (2,2 meses), sendo a mediana de 59 dias (1,9 mês), o que sugere que grande parte delas já teria o nome definido pela família. Este campo, portanto, já deveria ter sido preenchido pelos profissionais de saúde da UBS nas diversas oportunidades de contato com a criança, por ocasião das ações da Primeira Semana de Saúde Integral, vacinação e atendimento pela equipe $e^{(2,3)}$.

Os dados referentes ao endereço também foram pouco preenchidos. Interessante considerar que, na lógica da organização do sistema de saúde, o local de moradia tem importância fundamental. Ele vincula o indivíduo à área de abrangência de um determinado serviço e equipe de saúde, além de informar, de maneira geral, níveis de risco socioambiental. Endereço e nome garantem a possibilidade de recuperação do documento, em caso de perda ou extravio. CSC sem nome e endereço sugere sua pouca valorização, não só por parte dos profissionais que a manuseiam, mas também por parte da família da criança.

Como era de se esperar, o número do cartão do SUS foi preenchido por apenas $3 \%$, pois ele ainda não está implantado em toda a cidade de Belo Horizonte. Com relação ao registro civil (2\% de preenchimento), supondo que grande parte das crianças não foi registrada ao sair da maternidade, o dado deveria ter sido completado pelos profissionais da UBS.

Nas informações sobre gravidez, parto e puerpério, verificou-se o baixo preenchimento de todos os campos. O início do pré-natal e o número de consultas são informações essenciais para avaliar a assistência à gestante e as condições de desenvolvimento da criança no útero. O Ministério da Saúde recomenda, para as gestantes de risco habitual, seis consultas durante a gravidez ${ }^{(4)}$. Em respectivamente 40 e $31 \%$ das CSC estudadas, os campos referentes ao início do pré-natal e número de consultas foram deixados em branco. Informações sobre condições maternas que interferem na saúde do feto, como grupo sanguíneo, fator Rh e tipo de gravidez, não foram preenchidas em proporção próxima a um terço das cadernetas.
O diagnóstico precoce da infecção pelo vírus da imunodeficiência humana (HIV), sífilis, hepatite B e toxoplasmose nas mulheres grávidas possibilita intervenções precoces, no sentido de prevenir ou reduzir danos à mãe e à criança. Assim, no pré-natal, a realização de sorologia para detecção destas infecções está indicada para todas as gestantes ${ }^{(4)}$. Entretanto, nas CSC estudadas, os campos para registro da sorologia materna para HIV, sífilis, hepatite B e toxoplasmose foram preenchidos em aproximadamente metade delas. Estes campos referem-se aos agravos codificados pelo CID-10, o que torna o preenchimento complexo e informam apenas se a sorologia foi realizada ou não e se a conduta foi instituída ou não. Os campos não informam o resultado propriamente dito do exame e não qualificam a conduta instituída, que são os dados que mais interessam. Isso poderia explicar em parte a omissão de seu adequado preenchimento.

Os campos destinados ao registro da vacinação da gestante com a dupla adulto, suplementação com ferro e administração de vitamina A no pós-parto estavam preenchidos em menos de $40 \%$ dos casos. Estes procedimentos são indicados de rotina, sendo a aplicação de megadose de vitamina A no puerpério reservada para as moradoras das áreas de risco de hipovitaminose $A^{(5,6)}$.

Em relação às informações sobre as condições do recémnascido, a situação do preenchimento das CSC é um pouco melhor. Peso ao nascer, comprimento e perímetro cefálico foram informados em respectivamente 91, 88 e $85 \%$ das cadernetas. Já as informações sobre certas condições de nascimento como Apgar, necessidade de reanimação e idade gestacional foram registradas em menor proporção $(74,71$ e 75\%, respectivamente). Na França, onde há 50 anos é adotado o Carnet de Santé de l'Enfant, foi realizado um estudo transversal multicêntrico de 1.680 crianças para avaliar a capacidade de informação do instrumento. Os valores do escore de Apgar no primeiro e quinto minuto de vida foram escolhidos como indicadores do estado de saúde ao nascimento, entre outros. Verificou-se o registro de pelo menos um escore de Apgar, do primeiro ou quinto minuto, em $96 \%$ das cadernetas francesas ${ }^{(7)}$.

Grupo sanguíneo e fator $\mathrm{Rh}$ do recém-nascido, bem como a realização do teste de Coombs, foram preenchidos em menos de $15 \%$ dos casos. Provavelmente, estes dados foram menos valorizados porque a chance de incompatibilidade sanguínea materno-fetal é baixa para a maioria dos recém-nascidos, especialmente na ausência de icterícia precoce.

O campo referente à triagem auditiva neonatal (teste da orelhinha) foi deixado em branco em mais da metade das 
cadernetas. A triagem auditiva neonatal universal tem sido amplamente recomendada e, no Brasil, os programas ainda estão em fase de implantação ${ }^{(8)}$. Em Belo Horizonte, apenas dois hospitais públicos realizam a triagem auditiva neonatal de rotina, embora, no município, o procedimento já esteja regulamentado por lei municipal. O baixo índice de preenchimento deste campo na CSC poderia, em parte, ser atribuído à pouca familiarização dos profissionais de saúde com esta prática.

O tipo de alimentação do recém-nascido, por ocasião da alta da maternidade, deixou de ser informado em $42 \%$ dos casos. O aleitamento materno exclusivo deve ser iniciado no pós-parto imediato, conforme preconiza a Organização Mundial de Saúde (OMS) em "Dez passos para o sucesso do aleitamento materno"(9). Estudo realizado em Belo Horizonte, entre 1980 e 2004, demonstrou que as mães cujos recém-nascidos não iniciaram a amamentação na maternidade tiveram risco duas vezes maior de desmamar do que aqueles que receberam leite materno logo após o parto ${ }^{(10)}$. O registro na CSC da alimentação, por ocasião da alta da maternidade, tem, portanto, fundamental importância para a continuidade da assistência ao binômio mãe-filho na atenção básica, pois permite a imediata intervenção da equipe de saúde para prevenir o desmame precoce.

As orientações para as ações da Primeira Semana de Saúde Integral estão na primeira folha da CSC. Assim, na maternidade, o profissional deverá orientar a mãe e anotar o dia e o local para a realizar as ações preconizadas: avaliação da mãe e do recém-nascido, com atenção especial à saúde mental da puérpera; orientações e apoio ao aleitamento materno; vacinação da mãe e recém-nascido; teste do pezinho; orientação para contracepção; agendamento de consulta para mãe e recém-nascido ${ }^{(2,11)}$. Em $75 \%$ das cadernetas, verificou-se que este campo foi deixado em branco, o que sugere a frágil integração entre os níveis de atenção hospitalar e ambulatorial. Mesmo considerando que as CSC distribuídas pelas maternidades públicas apresentam uma proporção significativamente maior de registro neste campo, esta proporção ainda é bem distante da esperada.

Mais de dois terços das mães afirmaram não ter recebido explicações sobre a CSC na maternidade. A compreensão pela família da função deste instrumento no acompanhamento da saúde infantil é essencial para que ela dele se aproprie, valorize-o e cobre do profissional o seu adequado preenchimento. Por outro lado, a falta de preenchimento dos campos bem como a ausência de explicações sobre os registros efetuados sugerem à família a pouca importância do instrumento no acompanhamento da sua criança. Assim, num círculo vicio- so, as cadernetas não são valorizadas pelas famílias: não são levadas às UBS nas consultas de rotina, são mal conservadas e, sobretudo, perdem seu caráter de documento de saúde. Quando interrogadas sobre a função da caderneta, dentre aquelas mães que responderam que "não serve para nada", verificou-se que uma proporção elevada não havia recebido explicações na maternidade, sugerindo a importância do diálogo entre profissional de saúde e família sobre este instrumento.

Por outro lado, é interessante notar que, a despeito da falta de explicações na maternidade, as mães atribuem múltiplas funções para a caderneta. Presume-se que, em outras oportunidades, como nas consultas de rotina, nas conversas com outras mães, na mídia e na própria experiência com o cuidado de filhos anteriores, as entrevistadas tenham elaborado suas percepções acerca das funções da CSC. Nota-se que a metade delas refere-se à função de registro de acompanhamento e $45 \%$ à função de cartão de vacina, ao qual tradicionalmente estão mais familiarizadas.

Esta pesquisa permite concluir que a CSC não tem sido utilizada adequadamente. Todavia, a interpretação destes resultados deve ser feita com cautela, pois o estudo apresenta limitações. Embora o número de cadernetas estudadas tenha sido elevado, não se tratou de amostra randomizada. Porém, o fato de o estudo ter sido conduzido em 29 UBS com crianças nascidas em 17 maternidades pode certamente espelhar a situação de um grupo bastante representativo. Outro aspecto a ser considerado é que os resultados podem não refletir as práticas do preenchimento das cadernetas nas maternidades, uma vez que, mesmo para itens de sua responsabilidade, os campos acabam sendo preenchidos nas UBS. Assim, o registro de dados da gravidez, parto e recém-nascido, supostamente realizado pelas maternidades, acaba por ser superestimado, englobando registros efetuados nas UBS.

No Brasil, os raros estudos que avaliam o Cartão da Criança, antecessor da CSC, apontam falhas consideráveis na sua utilização. Em Feira de Santana, Bahia, estudo envolvendo 2.319 menores de um ano evidenciou ausência de registro do Apgar em $72 \%$ dos cartões, falhas no registro do peso em $34 \%$ e falhas no registro dos marcos de desenvolvimento em $78 \%{ }^{(12)}$. Em Pernambuco, pesquisa com 1.669 crianças mostrou que $13 \%$ não tinham o registro do peso de nascimento no cartão ${ }^{(13)}$. Em Belém, Pará, estudo com 560 crianças mostrou que $73 \%$ portavam o Cartão da Criança, nos quais apenas $12 \%$ contavam com informações do nascimento completamente preenchidas $^{(14)}$. Em Teresópolis, Rio de Janeiro, pesquisa com 329 crianças menores de um ano mostrou que mais de $90 \%$ delas havia comparecido à consulta pediátrica 
nos três meses anteriores e quase todas possuíam o Cartão da Criança, embora não houvesse qualquer registro de peso neste período em $30 \%$ destes cartões ${ }^{(15)}$.

A precariedade do preenchimento dos dados sobre gravidez, parto e recém-nascido nas CSC estudadas leva a uma reflexão sobre as condições de trabalho dos profissionais de saúde, bem como sobre a sua falta de preparo para lidar com este instrumento. A rotina do trabalho nas maternidades é estressante e, muitas vezes, o médico e o enfermeiro ficam sobrecarregados. Além do universo da assistência, o trabalho envolve o preenchimento de inúmeros formulários e procedimentos burocráticos demandados pela instituição. Se os próprios profissionais não estão sensibilizados para a importância da CSC, ela passa a ser mais um formulário a ser preenchido. Outro aspecto a se considerar é que não está claramente definido a qual profissional compete o preenchimento da CSC nas maternidades, o que leva à diluição de responsabilidades e dificuldades nos esforços de capacitação e avaliação.

\section{Referências bibliográficas}

1. Brasil - Ministério da Saúde. Secretaria de Atenção à Saúde - Departamento de Ações Programáticas Estratégicas. Manual para utilização da Caderneta de Saúde da Criança. Brasília: Ministério da Saúde, 2005.

2. Brasil - Ministério da Saúde. Secretaria de Atenção à Saúde - Departamento de Ações Programáticas Estratégicas. Pacto nacional pela redução da mortalidade materna e neonatal. Brasília: Ministério da Saúde, 2004.

3. Minas Gerais - Secretaria de Estado da Saúde. Atenção ao pré-natal, parto e puerpério: protocolo Viva Vida. $2^{\mathrm{a}}$ ed. Belo Horizonte: SAS/SES, 2006.

4. Brasil - Ministério da Saúde. Secretaria de Atenção à Saúde - Departamento de Ações Programáticas Estratégicas. Pré-natal e puerpério: atenção qualificada e humanizada.

Brasília: Ministério da Saúde, 2005.

5. Brasil - Ministério da Saúde. Secretaria de Atenção à Saúde - Departamento de Ações Básicas. Saúde de ferro: programa nacional de suplementação de ferro. Brasília: Ministério da Saúde, 2005.

6. Brasil - Ministério da Saúde. Vitamina A. Departamento de Atenção Básica, Alimentação e Nutrição. Programa nacional de suplementação da vitamina A: condutas gerais [monograph on the Internet]. Brasília: Ministério da Saúde; 2004 [cited 2007 Nov 21]. Available from: http://dtr2004.saude. gov.br/nutricao/vita.php

7. Vincelet C, Tabone MD, Berthier M, Bonnefoi MC, Chevalier B, Lemaire JP; Group de Pédiatrie Générale de la Société Française de Pédiatrie. How are personal child health records completed? A multicentric evaluation study. Arch Pediatr 2003;10:403-9.

8. Tiensoli LO, Goulart LM, Resende LM, Colosimo EA. Hearing screening in a public hospital in Belo Horizonte, Minas Gerais State, Brazil: hearing
Atualmente, a CSC está na terceira versão, bem mais completa do que a anterior, o que amplia as suas possibilidades enquanto instrumento de vigilância e educação. Todavia, garantir a sua plena utilização é um desafio ainda mais difícil. Faz-se, portanto, necessário um trabalho intensivo de divulgação, sensibilização e capacitação de todos os profissionais envolvidos com a saúde materno-infantil para que a caderneta possa efetivamente ser utilizada para a promoção da saúde da criança.

\section{Agradecimentos}

Agradecemos à Secretaria Municipal de Saúde de Belo Horizonte e ao Núcleo de Estudos em Saúde Coletiva e Nutrição da Faculdade de Medicina da Universidade Federal de Minas Gerais (UFMG) pelo apoio. Aos alunos do Curso de Especialização em Saúde da Família BHVida, os nossos agradecimentos pela efetiva participação neste trabalho. impairment and risk factors in neonates and infants. Cad Saude Publica 2007;23:1431-41.

9. Brasil - Ministério da Saúde. Organização Pan-Americana de Saúde. Dez passos para uma alimentação saudável: guia alimentar para crianças menores de dois anos [monograph on the Internet]. Brasília: Ministério da Saúde; 2002 [cited 2007 Apr 5]. Available from: http://bvsms.saude.gov.br/bvs/publicacoes/10_passos.pdf

10. Alves, CRL. Aleitamento materno no Centro de Saúde São Marcos (Belo Horizonte/MG): lições de uma história de 25 anos [tese de doutorado]. Belo Horizonte (MG): Faculdade de Medicina da UFMG; 2005.

11. Coordenação de Atenção à Criança - Gerência de Assistência - Secretaria Municipal de Saúde - Prefeitura de Belo Horizonte. Agenda de compromissos pela saúde integral da criança e adolescente e redução da mortalidade infantil [monograph on the Internet]. Belo Horizonte: Secretaria Municipal de Saúde; 2004 [cited 2007 Apr 5]. Available from: http://www.pbh.gov.br/smsa/ biblioteca/geas/agendadacrianca.pdf

12. Vieira GO, Vieira TO, Costa MCO, Netto PVS, Cabral VA. Uso do cartão da criança em Feira de Santana, Bahia. Rev Bras Saúde Mater Infant 2005;5:177-184.

13. Ratis CAS, Batista Filho M. Aspectos estruturais e processuais da vigilância do crescimento de menores de cinco anos em serviços públicos de saúde do Estado de Pernambuco. Rev Bras Epidemiol 2004;7:44-53.

14. Figueiras ACM, Barros LCN, Barléte KCS, Faria ECF, Fernandes MSB, Santos JRP. Uso do cartão da criança no município de Belém. Rev Paraense Med 2001;15:39.

15. Santos SR, Cunha AJ, Gamba CM, Machado FG, Leal Filho JM, Moreira NLM. Avaliação da assistência à saúde da mulher e da criança em localidade urbana da Região Sudeste do Brasil. Rev Saude Publica 2000;34:266-71. 